\title{
Decision Support System for Exemplary Teacher Selection in Elementary School using Topsis Method
}

\author{
Rara Pramita Putri, M. Ilayaraja, K. Shankar, Wahidah Hashim, Irina N. Odarich, Andino \\ Maseleno
}

\begin{abstract}
Teacher is one of the most important components owned by school in sustaining life. It is a challenge for the management of educational institutions to be able to provide an appropriate, effective and efficient decision in data management in the competitive business world. It spurs schools to strive harder in improving the quality of the school. Technique for Order Preference by Similarity to Ideal Solution (TOPSIS) is a method that has a concept where the best selected alternative not only has the shortest distance from a ideal positive solution. But also has the longest distance from a ideal negative solution. The criteria used in this system vary, according to the selection of the best teachers provided by the institution. The applicant's score data that has been entered into the system will be calculated using TOPSIS method, by finding the farthest and closest distance from the positive and ideal negative solutions. Applicants with the highest $v$ score will occupy the top of this system. Based on the results of the sample case selection shows that the results of calculations using the same system with manual calculations. This system is able to provide the best teacher recommendations.
\end{abstract}

Keywords : Best Teacher, DSS, TOPSIS.

\section{INTRODUCTION}

\section{A. Background of Problem}

$\mathrm{D}_{\mathrm{e}}$ ecision support system is interactive information system that has provided information, modeling, and data manipulation. The system used has function to help decisions maker in semi-structured situation and unstructured situation, where nobody knows exactly how a decision must have been made. Decision support system usually made for finding a solution or some problem or to evaluate some opportunity. Decision support system doesn't intend to automate decision making, but gives interactive device that let decision maker do various analysis with using available models [7].

The role of a teacher in the progress of education process is very important. Teachers is one of the key factor for the

Revised Manuscript Received on December 16, 2019.

* Correspondence Author

Rara Pramita Putri, Department of Information System, STMIK Pringsewu, Lampung, Indonesia

M. Ilayaraja*, School of Computing, Kalasalingam Academy of Research and Education, Krishnankoil, Tamilnadu, India. Email: ilayaraja.m@klu.ac.in

K. Shankar, School of Computing, Kalasalingam Academy of Research and Education, Krishnankoil, Tamilnadu, India.

Wahidah Hashim, Institute of Informatics and Computing Energy, Universiti Tenaga Nasional, Malaysia.

Andino Maseleno, Institute of Informatics and Computing Energy, Universiti Tenaga Nasional, Malaysia.

Irina N. Odarich, Togliatti State University, Russia. creation of high quality future generations with the main task of educating, teaching, guiding, directing, and so on. Research that conducted with the title Decision Support System for Exemplary Teacher Selection at SDN 3 Pujojadi Using TOPSIS Method conducted to made a recommendation for exemplary teachers at SDN 3 Pujodadi [4].

Nowadays, the utilization of science and technology develops very quickly and produces new innovations that must be balanced with the ability to adapt to the technology. One of them is the decision support system for the exemplary teacher selection at SDN 3 Pujodadi. In the exemplary teacher selection we can do some research on the aspect of performance or its competence.

Exemplary teacher selection is expected for motivating teacher to be active in doing its activities and which is expected to have positive effect on its work to face challenges in this globalization era.

In conducting exemplary teacher selection we can organize professionally or objectively which means the selection is done competitively for their abilities and skills and work potential, not based on equitable opportunities for participate the example teacher's selection so that the selection process can be more structured, precise, and easy to do.

In addition, the process of monitoring the performance of teachers will be easier to do by the school and follow up with the evaluation process or other planning that related to the performance of the teacher. With the information system, monitoring and evaluation of teachers performance based on the assessment of the TOPSIS method will help the SDN 3 Pujodadi to implement more objective electoral process. [1]

\section{B. Problem Formulation}

In this formulation of problem how to make decision support system that can help to decide exemplary teacher selection:

- How to apply the criteria and abilityto determine exemplary teacher.

- How to apply the TOPSIS method on the decision support system todetermine the exemplary teacher at SDN 3 PUJODADI.

- How to design a decision support system application to determine exemplary teacher selection.

Published By: 


\section{Objectives}

There are some objectives of research from exemplary teacher selection at SDN 3 Pujodadi as follows:

- Improving teacher motivation in carrying out their duties.

- Improving elementary school teacher competition in the selection of these exemplary teachers.

- Applying criteria and weight in exemplary teachers selection at SDN 3 PUJODADI.

- Creating a decision support system for the exemplary teachers selection with structured data that can be accessed quickly or accurately.

\section{LITERATURE REVIEW}

\section{A. Decision Support System}

According to [16], DSS is the process concluded that the decision is assisted using a computer to assist with the return of the decision by using some data.

DSS is an interactive system, helping decision-making through the use of data and decision models to solve problems that are semi-structured and unstructured [8].

DSS is a computer-based system that makes various alternative decisions to help various management in response to various problems that are structured or not with using data and models [12].

From the opinion given above, it can be concluded that the Decision Support System (DSS) is a computer-based system to help management in handling various problems structured or unstructured with using data and models. The criteria used in determining the exemplary teacher's selection is performance certificate, education level, work discipline, work quality, and length of work. The decision support system is a system that capable to provide problem solving capabilities and communication skills for problems with semi-structured and unstructured situations. This system is used to help decision making in situations semi-structured and unstructured situations, where no one knows exactly how the decision should have been made [17].

\section{B. TOPSIS Method}

TOPSIS is one of the multi criteria or alternative choice decision making methods which is an alternative that has the smallest distance from the ideal positive solution and the largest distance from the ideal negative solution from a geometric point of view with using a distance of Euclidean [11] - [14]. However, alternatives that have the smallest distance from an ideal positive solution, don't have to have the largest distance from the ideal negative solution. Therefore, TOPSIS consider both, the distance to ideal positive solution and the distance to the ideal negative solution simultaneously [15]. The optimal solution in the TOPSIS method is obtained by determining the relative proximity of an alternative to the ideal positive solution. TOPSIS will rank the alternative based on the relative proximity score priority of an alternative to the ideal positive solution. The alternatives that have been ranked are used as a reference for decision makers to choose the best solution that is desired. The TOPSIS method is one of the Fuzzy Multiple Attribute Decision Making (FMADM) Method which is a method used to find the optimal alternatives of a number of alternatives with certain criteria.

\section{Teacher}

Based on Republic Indonesia Law No 14 Year 2005, teachers are professional educators with the primary task of educating, teaching, guiding, directing, training, assessing, and evaluating students on early childhood education, formal education ways, primary education, and secondary education. The teacher is a professional educator because implicitly he has made himself accepted and assumed some of his educational responsibilities on the shoulders of the parents [7] [3], [8]-[10].

\section{Characteristic of Exemplary Teacher}

- The teacher must have a clean Aqidah from the things that contradict with that.

- Be able to regulate time well, so that there is no time to miss without get the benefit of worldly and Ukhrawi

- The teacher must be an example of the students in all words, deeds and attitudes.

- Teachers must always be honest, fair, say good things, and give advice and guide to thestudents.

\section{E. SD Negeri 3 Pujojadi}

Public elementary School (SDN) 3 Pujodadi is one of basic level of formal education in Indonesia. In determining the teacher performance criteria in SD Negeri 3 Pujodadi still use manual way. SD Negeri 3 Pujodadi is an elementary school organized by both government and private sector. The management of Public elementary School (SDN) which is previously under the Education Office, especially at the Pujodadi district of Pringsewu. SD Negeri 3 Pujodadi was established in 1980 by the government.

\section{F. Elementary School Teacher}

Teacher is an educator, who is a role model, in the identification for the students and the environment. The teacher also has many tasks, both bound by the office and outside the service, in the form of devotion. Therefore, in determining the exemplary teacher selection at SDN 3 Pujodadi using the TOPSIS method to the needs of the criteria and weights to conduct the assessment so that it will be easy to do the election.

\section{THE METHOD OF RESEARCH}

\section{A. Data Collection}

In this method, writer used data collection method that would be used to fulfill need of application. Method that used in data collection as follows:

\section{Interview Method}

It is a conversation between researchers and informers, researchers here are hoping to get the interview information done directly to the teachers related to the electoral system.

\section{Observation Method}

It is a research method where researchers observe all the activities found in the field, in order to support the interview data with the intention to provide solutions through the information system to be built so that it is easier to do an observation.

\section{Literature Method}

Literature method is a data collection technique by studying the reference of documents or files and the collection of data on legislation, research

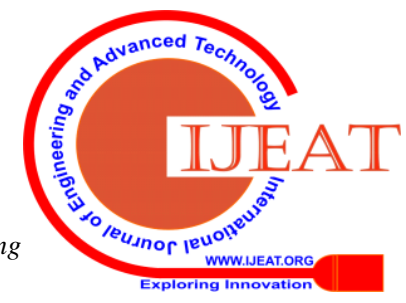


journals through literature review conducted a study of legislation that related with management of regional potential. Data need that reveals about the indicators used.

\section{B. TOPSIS Method}

TOPSIS is one of the multi criteria or alternative choice decision making methods which is an alternative that has the smallest distance from the ideal positive solution and the largest distance from the ideal negative solution from a geometric point of view with using a distance of Euclidean [11] - [14]. However, alternatives that have the smallest distance from an ideal positive solution don't have to have the largest distance from the ideal negative solution. Therefore, TOPSIS consider both, the distance to ideal positive solution and the distance to the ideal negative solution simultaneously [15]. The optimal solution in the TOPSIS method is obtained by determining the relative proximity of an alternative to the ideal positive solution. TOPSIS will rank the alternative based on the relative proximity score priority of an alternative to the ideal positive solution. The alternatives that have been ranked are used as a reference for decision makers to choose the best solution that is desired. The TOPSIS method is one of the Fuzzy Multiple Attribute Decision Making Method (FMADM) which is a method used to find the optimal alternatives of a number of alternatives with certain criteria.

Some of TOPSIS procedures are:

- Create a normalized decision matrix

- Create a weighted, normalized decision matrix

- Determine the ideal matrix of positive solutions and the ideal matrix of negative solutions

- Determine the distance between alternative scores with the matrix of the ideal positive solution and matrix of very negative solutions

- Determine the preference score for each alternative

TOPSIS requires the performance rating of each AI alternative in each of the normalizedCJ criteria:

$$
r_{i j=\frac{x_{i j}}{\sum_{i}^{m}=1_{i j}^{x^{2}}}}
$$

Where:

$$
\begin{aligned}
& r_{i j=} \text { the result of normalizing thedecision matrix } \mathrm{R} \\
& \mathrm{I}=1,2 \ldots, \mathrm{m} \\
& \mathrm{J}=1,2 \ldots, \mathrm{n}
\end{aligned}
$$

Ideal positive solution $A^{+}$and ideal negative solution $A^{-}$can be determined with normalized weight rating $\left(y_{i j}\right)$ as follows:

$$
y_{i j}=W_{i} 1_{i j}
$$

With $1=1,2 \ldots . \mathrm{m}$, and $\mathrm{j}=1,2 \ldots \mathrm{n}$

$$
\begin{aligned}
& A^{+}=\left(y_{1}^{+} \cdot y_{2}^{+} \ldots \ldots y_{n}^{+}\right) . \\
& A^{-}=\left(y_{1}^{-} \cdot y_{2}^{-} \ldots \ldots \ldots y_{n}^{-}\right) .
\end{aligned}
$$

The steps of this TOPSIS method are as follows:

1. TOPSIS begins by building a decision matrix, the $\mathrm{X}$ decision matrix refers to the malternative which will be evaluated based on the criteria.

$$
x=\left\{\begin{array}{c}
A_{1} X_{11} X_{12} X_{13} \ldots . X_{1 n} \\
A_{2} X_{11} X_{22} X_{23} \ldots . X_{2 n} \\
A_{3} X_{31} X_{32} X_{33} \ldots . X_{3 n} \\
A_{m} X_{m 1} X_{m 3} X_{m 3} \ldots . X m m
\end{array}\right.
$$

Where $\mathrm{Ai}(\mathrm{i}=1,2,3, \ldots \mathrm{m})$ is the possible alternative, $\mathrm{Xj}$ $(j=1,2,3, \ldots . . n)$ is attribute where an alternate performance is measured, Xij is an alternate Ai performance with the reference to the $\mathrm{Xj}$ attribute.

2. Create a normalized decision matrix.

$$
\begin{gathered}
r i j=\frac{x i j}{\sqrt{\sum_{i=1}^{m} x_{i j}^{2}}} \\
\text { with } \mathrm{i}=1,2, \ldots, \mathrm{m} \\
\mathrm{j}=1,2, \ldots, \mathrm{n} \\
\text { where : } \\
\mathrm{r}_{\mathrm{ij}}=\text { normalized matrix }[\mathrm{i}][\mathrm{j}] \\
\mathrm{X}_{\mathrm{ij}}=\text { decision matrix }[\mathrm{i}][\mathrm{j}]
\end{gathered}
$$

3. Create normalized, weighted matrix decision

$V_{i j}=w_{i} r_{i j}$;

with $\mathrm{i}=1,2, \ldots, \mathrm{m}$; and $\mathrm{j}=1,2, \ldots, \mathrm{n}$

Where :

$\mathrm{Vij}=$ Elements of a weighted normalized decision matrix $\mathrm{V}$ wi $=$ jth criteria weight

rij $=$ Normalized decision matrix elements $\mathrm{R}$

4. Ideal positive solution $\mathrm{A}+$ and ideal negative solution Acan be determined based on normalized weight rating (yij) as: $\mathrm{A}^{+}=\left(\mathrm{y}_{1}^{+}, \mathrm{y}_{2}^{+}, \ldots, \mathrm{y}_{\mathrm{n}}^{+}\right)$;

$\mathrm{A}^{-}=\left(\mathrm{y}_{1}^{-}, \mathrm{y}_{2}^{-}, . ., \mathrm{y}_{\mathrm{n}}^{-}\right)$;

Where :

$$
\mathrm{V}_{\mathrm{j}}^{+}=\max \mathrm{Y}_{\mathrm{ij}} \mathrm{j} \text { is benefit attribute }
$$

Min Yij if $\mathrm{j}$ is cost attribute

$$
\mathrm{V}_{\mathrm{j}}^{-}=\min \mathrm{yij} \text {, if } \mathrm{j} \text { is benefit attribute }
$$

$\max y i j$, if $j$ is cost attribute

5. DistanceAi alternative with ideal positive solutions:

$\left.D_{i}^{+}=\sqrt{\sum_{i-1}^{n}\left(Y_{i}^{+}\right.}-Y_{i j}\right)^{2}$

Where :

$\mathrm{D}_{\mathrm{i}}^{+}=$Distance Ai alternative with ideal positive solutions

$$
\mathrm{Y}_{\mathrm{j}}^{+}=\text {Ideal positive solutions [i] }
$$

Yij = normalized matrix $[\mathrm{i}][\mathrm{j}]$

6. Distance between $\mathrm{Ai}$ alternative and ideal negative solution

$\left.D_{i}^{-}=\sqrt{\sum_{i-1}^{n}\left(Y_{i j^{-}}\right.} Y_{i}^{-}\right)^{2}$

$\mathrm{i}=1,2, \ldots \mathrm{m}$

Where :

$\mathrm{D}_{\mathrm{i}}^{-}=$Distance Ai alternative with ideal negative solutions

$\mathrm{Y}_{\mathrm{j}}^{-}=$ideal negative solution [i] 


\section{Decision Support System for Exemplary Teacher Selection in Elementary School using Topsis Method}

Yij = normalized matrix $[\mathrm{i}][\mathrm{j}]$

7. The preference score for each alternative ( $\mathrm{Vi})$ is given as:

$V_{i}=\frac{D_{i}^{-}}{D_{i}^{-}+D_{i}^{+}}$

$\mathrm{i}=1,2, \ldots \mathrm{m}$

$\mathrm{Vi}=$ Proximity of each alternative to the ideal

solution

$\mathrm{D}_{\mathrm{i}}^{+}=$Distance between Ai alternative and ideal

positive solutions

$\mathrm{D}_{\mathrm{i}}^{-}=$Distance between Ai alternativeand ideal negative solutions

A larger score of $\mathrm{Vi}$ indicates that an alternative $\mathrm{Ai}$ was preferred.

\section{Method Criteria}

In this research there are criteria for determine teacher's performance assessment

Table-I: Criteria

\begin{tabular}{|l|l|}
\hline \multicolumn{1}{|c|}{ Code } & \multicolumn{1}{c|}{ Description } \\
\hline C1 & Performance certificate \\
\hline C2 & Education level \\
\hline C3 & Work discipline \\
\hline C4 & Work quality \\
\hline C5 & Length of work \\
\hline
\end{tabular}

\section{Criteria weight score}

This weight score determined based on the importance of these criteria

Table-II: Criteria weight score

\begin{tabular}{|c|l|c|}
\hline code & \multicolumn{1}{|c|}{ Description } & Weight score \\
\hline C1 & $\begin{array}{l}\text { Performance } \\
\text { certificate }\end{array}$ & $15 \%$ \\
\hline C2 & Education level & $25 \%$ \\
\hline C3 & Work discipline & $20 \%$ \\
\hline C4 & Work quality & $20 \%$ \\
\hline C5 & Length of work & $20 \%$ \\
\hline
\end{tabular}

\section{E. Manual Test}

Table-III: Certificate (C1)

\begin{tabular}{|l|c|}
\hline \multicolumn{1}{|c|}{ Performance Certificate } & Score \\
\hline Good enough & 1 \\
\hline Good & 3 \\
\hline Vey Good & 5 \\
\hline
\end{tabular}

Table-IV: Education level (C2)

\begin{tabular}{|l|c|}
\hline \multicolumn{1}{|c|}{ Compliance criteria } & Score \\
\hline SMP & 1 \\
\hline SMA & 2 \\
\hline D-III & 4 \\
\hline S1/S2 & 5 \\
\hline
\end{tabular}

Table-V: Work Discipline (C3)

\begin{tabular}{|l|c|}
\hline \multicolumn{1}{|c|}{ Discipline Criteria } & Score \\
\hline Very good & 5 \\
\hline Good & 3 \\
\hline
\end{tabular}

Table-VI: Work Quality(C4)

\begin{tabular}{|l|c|}
\hline \multicolumn{1}{|c|}{ Work quality criteria } & Score \\
\hline Good enough & 2 \\
\hline Good & 3 \\
\hline Very good & 5 \\
\hline
\end{tabular}

Published By:

\section{F. Flowchart}

The process in TOPSIS is the input of cadres performance weight per criterion

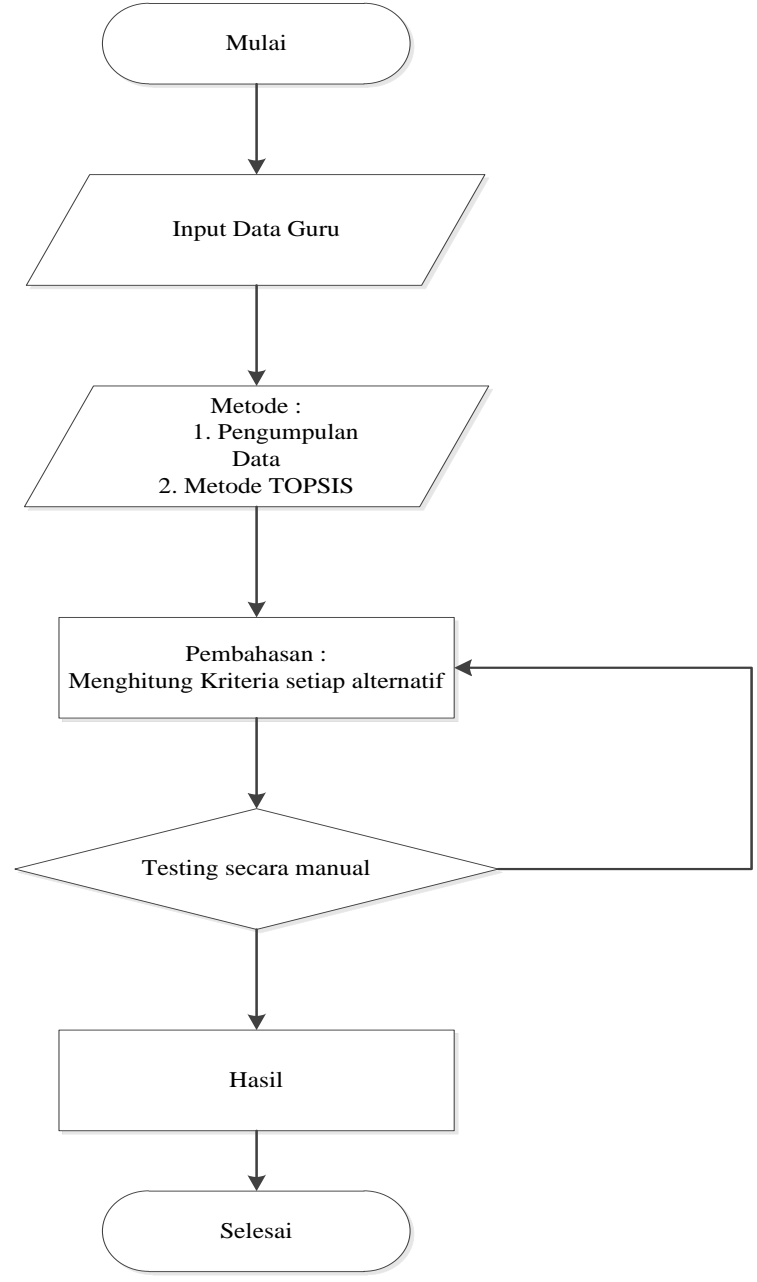

Fig. 1.Flowchart

Description:

1. In this research, data collection conducted so it will be easily understood

2. Determine criteria suitable with our research title used TOPSIS method

3. To make it easier for us to do the research, we collected the data according to what we research

4. At this stage we hadentered in the discussion to calculate, determining the criteria to alternatively perform calculations manually prefixed with the counters via Ms. Excel to make it easier and the result is the same as we manually calculated. 


\section{NUMERICAL EXAMPLE}

\section{A. Alternative Table}

Alternative table for all criteria:

\section{Table-VIII: Decision Matrix}

\begin{tabular}{|c|c|c|c|c|c|}
\hline Alternative & $\boldsymbol{C 1}$ & $\boldsymbol{C 2}$ & $\boldsymbol{C 3}$ & $\boldsymbol{C 4}$ & $\boldsymbol{C 5}$ \\
\hline A1 & 1 & 1 & 3 & 3 & 5 \\
\hline A2 & 3 & 2 & 5 & 2 & 4 \\
\hline A3 & 5 & 4 & 3 & 3 & 2 \\
\hline A4 & 1 & 5 & 3 & 5 & 1 \\
\hline A5 & 2 & 3 & 2 & 1 & 5 \\
\hline A6 & 3 & 3 & 5 & 1 & 2 \\
\hline A7 & 2 & 2 & 4 & 3 & 4 \\
\hline A8 & 1 & 2 & 4 & 3 & 2 \\
\hline A9 & 2 & 1 & 5 & 2 & 1 \\
\hline A10 & 2 & 3 & 3 & 5 & 2 \\
\hline
\end{tabular}

$\left|C_{1}\right|=\sqrt{1^{2}+3^{2}+5^{2}+1^{2}+2^{2}+3^{2}+2^{2}+1^{2}+2^{2}+2^{2}}$ $=\sqrt{62}=7.87400787$

$r_{11}=\frac{X_{11}}{X_{1}}=\frac{1}{7.87400787}=0.127000127$

$r_{21}=\frac{X_{21}}{X_{1}}=\frac{3}{7.87400787}=0.381000381$

$r_{31}=\frac{X_{31}}{X_{1}}=\frac{5}{7.87400787}=0.635000635$

$r_{41}=\frac{X_{41}}{X_{1}}=\frac{1}{7.87400787}=0.127000127$

$r_{51}=\frac{X_{51}}{X_{1}}=\frac{2}{7.87400787}=0.254000254$

$r_{61}=\frac{X_{61}}{X_{1}}=\frac{3}{7.87400787}=0.381000381$

$r_{71}=\frac{X_{71}}{X_{1}}=\frac{2}{7.87400787}=0.254000254$

$r_{81}=\frac{X_{81}}{X_{1}}=\frac{1}{7.87400787}=0.127000127$

$r_{91}=\frac{X_{91}}{X_{1}}=\frac{2}{7.87400787}=0.254000254$

$r_{10}=\frac{X_{10}}{X_{1}}=\frac{2}{7.87400787}=0.254000254$

$\left|C_{2}\right|=\sqrt{1^{2}+2^{2}+4^{2}+5^{2}+3^{2}+3^{2}+2^{2}+2^{2}+1^{2}+3^{2}}$

$=\sqrt{82}=9.05538514$

$r_{21}=\frac{X_{11}}{X_{1}}=\frac{1}{9.05538514}=0.110431526$

$r_{22}=\frac{X_{21}}{X_{1}}=\frac{2}{9.05538514}=0.220863052$

$r_{23}=\frac{X_{31}}{X_{1}}=\frac{4}{9.05538514}=0.441726104$

$r_{24}=\frac{X_{41}}{X_{1}}=\frac{5}{9.05538514}=0.55215763$

$r_{25}=\frac{X_{51}}{X_{1}}=\frac{3}{9.05538514}=0.331294578$

$r_{26}=\frac{X_{61}}{X_{1}}=\frac{3}{9.05538514}=0.331294578$

$r_{27}=\frac{X_{71}}{X_{1}}=\frac{2}{9.05538514}=0.220863052$

$r_{28}=\frac{X_{81}}{X_{1}}=\frac{2}{9.05538514}=0.220863052$

$r_{29}=\frac{X_{91}}{X_{1}}=\frac{1}{9.05538514}=0.110431526$

$r_{30}=\frac{X_{101}}{X_{1}}=\frac{3}{9.05538514}=0.331294578$

$\left|C_{3}\right|=\sqrt{3^{2}+5^{2}+3^{2}+3^{2}+2^{2}+5^{2}+4^{2}+4^{2}+5^{2}+3^{2}}$ $=\sqrt{147}=12.1243557$

$r_{31}=\frac{X_{11}}{X_{1}}=\frac{3}{12.1243557}=0.247435839$

$r_{32}=\frac{X_{21}}{X_{1}}=\frac{5}{12.1243557}=0.412393048$

$r_{33}=\frac{X_{31}}{X_{1}}=\frac{3}{12.1243557}=0.247435839$

$r_{34}=\frac{X_{41}}{X_{1}}=\frac{3}{12.1243557}=0.247435839$

$r_{35}=\frac{X_{51}}{X_{1}}=\frac{2}{12.1243557}=0.164957219$
$r_{36}=\frac{X_{61}}{X_{1}}=\frac{5}{12.1243557}=0.412393048$

$r_{37}=\frac{X_{71}}{X_{1}}=\frac{4}{12.1243557}=0.329914438$

$r_{38}=\frac{X_{81}}{X_{1}}=\frac{4}{12.1243557}=0.329914438$

$r_{39}=\frac{X_{91}}{X_{1}}=\frac{5}{12.1243557}=0.412393048$

$r_{40}=\frac{X_{101}}{X_{1}}=\frac{3}{12.1243557}=0.247435839$

$\left|C_{4}\right|=\sqrt{3^{2}+2^{2}+3^{2}+5^{2}+1^{2}+1^{2}+3^{2}+3^{2}+2^{2}+5^{2}}$ $=\sqrt{96}=9.79795897$

$r_{41}=\frac{X_{11}}{X_{1}}=\frac{3}{9.79795897}=0.306186218$

$r_{42}=\frac{X_{21}}{X_{1}}=\frac{2}{9.79795897}=0.204124145$

$r_{43}=\frac{X_{31}}{X_{1}}=\frac{3}{9.79795897}=0.306186218$

$r_{44}=\frac{X_{41}}{X_{1}}=\frac{5}{9.79795897}=0.510310363$

$r_{45}=\frac{X_{51}}{X_{1}}=\frac{1}{9.79795897}=0.102062073$

$r_{46}=\frac{X_{61}}{X_{1}}=\frac{1}{9.79795897}=0.102062073$

$r_{47}=\frac{X_{71}}{X_{1}}=\frac{3}{9.79795897}=0.306186218$

$r_{48}=\frac{X_{81}}{X_{1}}=\frac{3}{9.79795897}=0.306186218$

$r_{49}=\frac{X_{91}}{X_{1}}=\frac{2}{9.79795897}=0.204124145$

$r_{50}=\frac{X_{101}}{X_{1}}=\frac{5}{9.79795897}=0.510310363$

$\left|C_{5}\right|=\sqrt{5^{2}+4^{2}+2^{2}+1^{2}+5^{2}+2^{2}+4^{2}+2^{2}+1^{2}+2^{2}}$

$=\sqrt{100}=10$

$r_{51}=\frac{X_{11}}{X_{1}}=\frac{5}{10}=0.5$

$r_{52}=\frac{X_{21}}{X_{1}}=\frac{4}{10}=0.4$

$r_{53}=\frac{X_{31}}{X_{1}}=\frac{2}{10}=0.2$

$r_{54}=\frac{X_{41}}{X_{1}}=\frac{1}{10}=0.1$

$r_{55}=\frac{X_{51}}{X_{1}}=\frac{5}{10}=0.5$

$r_{56}=\frac{X_{61}}{X_{1}}=\frac{2}{10}=0.2$

$r_{57}=\frac{X_{71}}{X_{1}}=\frac{4}{10}=0.4$

$r_{58}=\frac{X_{81}}{X_{1}}=\frac{2}{10}=0.2$

$r_{59}=\frac{X_{91}}{X_{1}}=\frac{1}{10}=0.1$

$r_{60}=\frac{X_{101}}{X_{1}}=\frac{2}{10}=0.2$

From the above calculation is obtained by the normalized matrix (R):

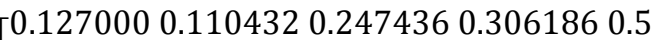
$\begin{array}{llll}0.381000 & 0.2208630 .4123930 .204124 & 0.4\end{array}$ 0.6350010 .4417260 .2474360 .3061860 .2 0.1270000 .5521580 .2474360 .510310 .1

$\mathrm{R}=$ 0.2540000 .3312950 .1649570 .1020620 .5 $\begin{array}{lllll}0.381000 & 0.3312950 .4123930 .102062 & 0.2\end{array}$ $\begin{array}{lllll}0.254000 & 0.220863 & 0.329914 & 0.306186 & 0.4\end{array}$ $\begin{array}{lllll}0.127000 & 0.220863 & 0.329914 & 0.306186 & 0.2\end{array}$

0.2540000 .1104320 .4123930 .2041240 .1

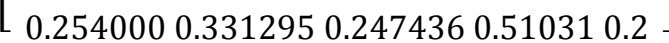

After obtained the normalized matrix (R), then look for matrix $\mathrm{V}$ based on the equation:

$V_{i j}=W_{j} X_{i j}$

$V_{1}=W_{1} r_{11}=(0,15)(0,127000)$

$V_{12}=W_{2} r_{12}=$ $=0,01905$

$(0,25)(0,110432)$ 


$$
\begin{aligned}
& =0,027608 \\
& V_{13}=W_{3} r_{13}=(0,20)(0,247436) \\
& =0,049487 \\
& V_{14}=W_{4} r_{14}=(0,20)(0,306186) \\
& =0,061237 \\
& V_{15}=W_{5} r_{15}=(0,20)(0,5) \\
& =0,1 \\
& V_{21}=W_{1} r_{21}=(0,15)(0,381000) \\
& =0,05715 \\
& V_{22}=W_{2} r_{22}=(0,25)(0,220863) \\
& =0,055216 \\
& V_{23}=W_{3} r_{23}=(0,20)(0,412393) \\
& =0,082479 \\
& V_{24}=W_{4} r_{24}=(0,20)(0,204124) \\
& =0,040825 \\
& V_{25}=W_{5} r_{25}=(0,20)(0,4) \\
& =0,08 \\
& V_{31}=W_{1} r_{31}=(0,15)(0,635001) \\
& =0,09525 \\
& V_{32}=W_{2} r_{32}=(0,25)(0,441726) \\
& =0,110432 \\
& V_{33}=W_{3} r_{33}=(0,20)(0,247436) \\
& =0,049487 \\
& V_{34}=W_{4} r_{34}=(0,20)(0,306186) \\
& =0,061237 \\
& V_{35}=W_{5} r_{35}=(0,20)(0,2) \\
& =0,04 \\
& V_{41}=W_{1} r_{41}=(0,15)(0,127000) \\
& =0,01905 \\
& V_{42}=W_{2} r_{42}=(0,25)(0,552158) \\
& =0,138039 \\
& V_{43}=W_{3} r_{43}=(0,20)(0,247436) \\
& =0,049487 \\
& V_{44}=W_{4} r_{44}=(0,20)(0,51031) \\
& =0,102062 \\
& V_{45}=W_{5} r_{45}=(0,20)(0,1) \\
& =0,02 \\
& V_{51}=W_{1} r_{51}=(0,15)(0,254000) \\
& =0,0381 \\
& V_{52}=W_{2} r_{52}=(0,25)(0,331295) \\
& =0,082824 \\
& V_{53}=W_{3} r_{53}=(0,20)(0,164957) \\
& =0,032991 \\
& V_{54}=W_{4} r_{54}=(0,20)(0,102062) \\
& =0,020412 \\
& V_{55}=W_{5} r_{55}=(0,20)(0,5) \\
& =0,1 \\
& V_{61}=W_{1} r_{61}=(0,15)(0,381000) \\
& =0,05715 \\
& V_{62}=W_{2} r_{62}=(0,25)(0,331295) \\
& =0,082824 \\
& V_{63}=W_{3} r_{63}=(0,20)(0,412393) \\
& =0,082479 \\
& V_{64}=W_{4} r_{64}=(0,20)(0,102062) \\
& =0,020412 \\
& V_{65}=W_{5} r_{65}=(0,20)(0,2) \\
& =0,04 \\
& V_{71}=W_{1} r_{71}=(0,15)(0,254000) \\
& =0,0381 \\
& V_{72}=W_{2} r_{72}=(0,25)(0,220863) \\
& =0,055216 \\
& V_{73}=W_{3} r_{73}=(0,20)(0,329914) \\
& =0,065983
\end{aligned}
$$

$$
\begin{aligned}
& V_{74}=W_{4} r_{74}=(0,20)(0,306186) \\
& =0,061237 \\
& V_{75}=W_{5} r_{75}=(0,20)(0,4) \\
& =0,08 \\
& V_{81}=W_{1} r_{81}=(0,15)(0,127000) \\
& =0,01905 \\
& V_{82}=W_{2} r_{82}=(0,25)(0,220863) \\
& =0,055216 \\
& V_{83}=W_{3} r_{83}=(0,20)(0,339914) \\
& =0,067983 \\
& V_{84}=W_{4} r_{84}=(0,20)(0,306186) \\
& =0,061237 \\
& V_{85}=W_{5} r_{85}=(0,20)(0,2) \\
& =0,04 \\
& V_{91}=W_{1} r_{91}=(0,15)(0,254000) \\
& =0,0381 \\
& V_{92}=W_{2} r_{92}=(0,25)(0,110432) \\
& =0,027608 \\
& V_{93}=W_{3} r_{93}=(0,20)(0,412393) \\
& =0,082479 \\
& V_{94}=W_{4} r_{94}=(0,20)(0,204124) \\
& =0,040825 \\
& V_{95}=W_{5} r_{95}=(0,20)(0,1) \\
& =0,02 \\
& V_{101}=W_{1} r_{101}=(0,15)(0,254000) \\
& =0,0381 \\
& V_{102}=W_{2} r_{102}=(0,25)(0,331295) \\
& =0,082824 \\
& V_{103}=W_{3} r_{103}=(0,20)(0,247436) \\
& =0,049487 \\
& V_{104}=W_{4} r_{104}=(0,20)(0,51031) \\
& =0,102062 \\
& V_{105}=W_{5} r_{105}=(0,20)(0,2) \\
& =0,04
\end{aligned}
$$

From the calculation above, obtained matrix Y:

$$
\mathrm{Y}=\left[\begin{array}{ccccc}
0.01905 & 0.027608 & 0.049487 & 0.061237 & 0.1 \\
0.05715 & 0.055216 & 0.082479 & 0.040825 & 0.08 \\
0.09525 & 0.110432 & 0.049487 & 0.061237 & 0.04 \\
0.01905 & 0.138039 & 0.049487 & 0.102062 & 0.02 \\
0.0381 & 0.082824 & 0.032991 & 0.020412 & 0.1 \\
0.05715 & 0.082824 & 0.082479 & 0.020412 & 0.04 \\
0.0381 & 0.055216 & 0.065983 & 0.061237 & 0.08 \\
0.01905 & 0.055216 & 0.067983 & 0.061237 & 0.04 \\
0.0381 & 0.027608 & 0.082479 & 0.040825 & 0.02 \\
0.0381 & 0.082824 & 0.049487 & 0.102062 & 0.04
\end{array}\right]
$$

Ideal positive solution calculated based on equation: $A^{+}=\left(y_{1}^{+} y_{2}^{+} \ldots y_{n}^{+}\right)$ $y_{1}^{+}=\max \left\{\begin{array}{c}0.01905 ; 0.05715 ; 0.09525 ; 0.01905 ; 0.0381 ; \\ 0.05715 ; 0.0381 ; 0.01905 ; 0.0381 ; 0.0381 ;\end{array}\right\}$ $=0.09525$

$y_{2}^{+}=$

$$
\begin{aligned}
& \max \left\{\begin{array}{l}
0.027608 ; 0.055216 ; 0.110432 ; 0.138039 ; 0.082824 ; \\
0.082824 ; 0.055216 ; 0.055216 ; 0.027608 ; 0.082824 ;
\end{array}\right\} \\
& =0.138039 \\
& y_{3}^{+}= \\
& \max \left\{\begin{array}{l}
0.049487 ; 0.082479 ; 0.049487 ; 0.049487 ; 0.032991 ; \\
0.082479 ; 0.065983 ; 0.067983 ; 0.082479 ; 0.049487 ;
\end{array}\right\} \\
& =0.082479 \\
& y_{4}^{+}= \\
& \max \left\{\begin{array}{l}
0.061237 ; 0.040825 ; 0.061237 ; 0.102062 ; 0.020412 ; \\
0.020412 ; 0.061237 ; 0.061237 ; 0.040825 ; 0.102062 ;
\end{array}\right\} \\
& =0.102062
\end{aligned}
$$


$y_{5}^{+}=\max \left\{\begin{array}{r}0.1 ; 0.08 ; 0.04 ; 0.02 ; 0.1 ; \\ 0.04 ; 0.08 ; 0.04 ; 0.02 ; 0.04 ;\end{array}\right\}$

$=0.1$

$A^{+}=\{0.09525 ; 0.138039 ; 0.082479 ; 0.102062 ; 0.1 ;\}$

Ideal negative solution calculated based on equation:

$A^{-}=\left(y_{1}^{-} y_{2}^{-} \ldots y_{n}^{-}\right)$

$y_{1}^{-}=\min \left\{\begin{array}{r}0.01905 ; 0.05715 ; 0.09525 ; 0.01905 ; 0.0381 ; \\ 0.05715 ; 0.0381 ; 0.01905 ; 0.0381 ; 0.0381 ;\end{array}\right\}$

$=0.0381$

$y_{2}^{-}=$

$\min \{0.027608 ; 0.055216 ; 0.110432 ; 0.138039 ; 0.082824 ;\}$

$\min \left\{\begin{array}{ll}0.027608 ; & 0.055216 ; 0.05216 ; 0.055216 ; 0.027608 ; 0.082824 ;\end{array}\right\}$

$=0.027608$

$y_{3}^{-}=$

$\min \{0.049487 ; 0.082479 ; 0.049487 ; 0.049487 ; 0.032991 ;\}=$ $\min \left\{\begin{array}{c}0.082479 ; 0.065983 ; 0.067983 ; 0.082479 ; 0.049487 ; \\ 0.082\end{array}\right\}=$ 0.032991

$y_{4}^{-}=$

$\min \{0.061237 ; 0.040825 ; 0.061237 ; 0.102062 ; 0.020412 ;\}$

$\min \left\{\begin{array}{l}0.020412 ; 0.061237 ; 0.061237 ; 0.040825 ; 0.102062 ;\end{array}\right.$ $=0.020412$

$y_{5}^{-}=\min \left\{\begin{array}{ccc}0.1 ; 0.08 ; 0.04 ; 0.02 ; 0.1 ; \\ 0.04 ; 0.08 ; 0.04 ; 0.02 ; 0.04 ;\end{array}\right\}$

$=0.02$

$A^{-}=\{0.0381 ; 0.027608 ; 0.032991 ; 0.020412 ; 0.02 ;\}$

The distance between the weighted score of each alternative to the ideal positive solution is calculated based on equation :

$D_{i}^{+}=\sqrt{\sum_{i=1}^{n}\left(Y_{i}^{+}-Y_{i j}\right)^{2}}$

$D_{1}^{+}=$

$\sqrt{\begin{array}{c}(0.01905-0.09525)^{2} \\ +(0.049487-0.082479)^{2} \\ +(0.1-0.1)^{2}\end{array}}$

$=\sqrt{0.35474}=0.59560$

$D_{2}^{+}=\sqrt{\begin{array}{c}(0.05715-0.09525)^{2}+(0.05521-0.13801)^{2} \\ +(0.08247-0.49487)^{2}+(0.04082-0.10206)^{2} \\ +(0.08-0.1)^{2}\end{array}}$

$=\sqrt{0.34705}=0.58910$

$D_{3}^{+}=$
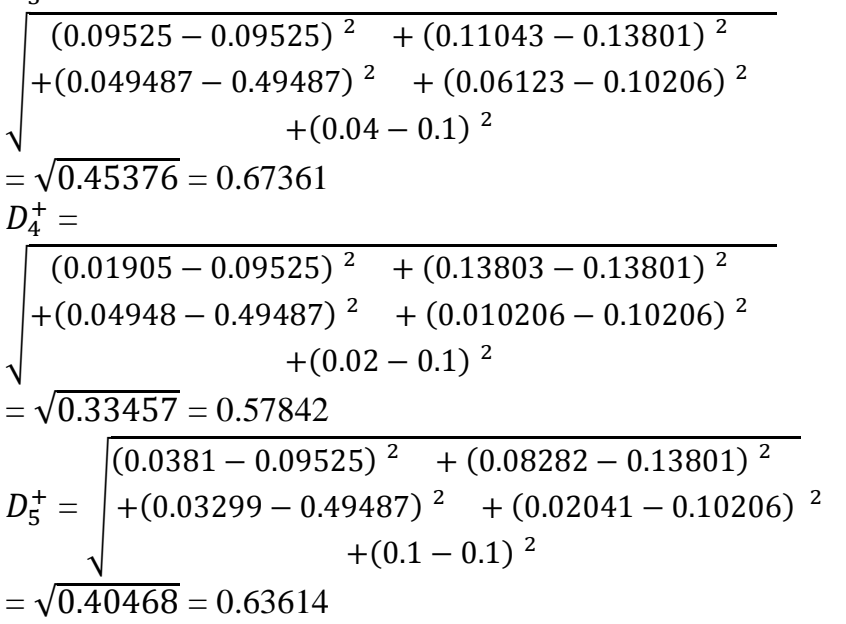

$$
\begin{aligned}
& D_{6}^{+}=\sqrt{\begin{array}{r}
(0.05715-0.09525)^{2} \\
+(0.08247-0.49487)^{2}+(0.08282-0.13801)^{2} \\
+(0.02041-0.10206)^{2} \\
+(0.04-0.1)^{2}
\end{array}} \\
& =\sqrt{0.31425}=0.56058
\end{aligned}
$$$$
\begin{aligned}
& D_{7}^{+}=\sqrt{\begin{array}{c}
(0.0381-0.09525)^{2}+(0.05521-0.13801)^{2} \\
+(0.06598-0.49487)^{2}+(0.06123-0.10206)^{2} \\
+(0.08-0.1)^{2}
\end{array}} \\
& =\sqrt{0.3649}=0.60406
\end{aligned}
$$

$$
\begin{aligned}
& D_{8}^{+}=\sqrt[\begin{array}{c}
(0.01905-0.09525)^{2}+(0.05521-0.13801)^{2} \\
+\left(0.06798-0.49487^{2}\right)+(0.06123-0.10206)^{2} \\
+(0.04-0.1)^{2}
\end{array}]{=\sqrt{0.30385}=0.55122} \\
& D_{9}^{+}=\sqrt[\begin{array}{c}
(0.0381-0.09525)^{2}+(0.02760-0.13801)^{2} \\
+(0.08247-0.49487)^{2}+(0.04082-0.10206)^{2} \\
+(0.02-0.1)^{2}
\end{array}]{ } \\
& =\sqrt{0.24039}=0.49029 \\
& D_{10}^{+}=\quad \begin{array}{c}
(0.03675-0.09525)^{2}+(0.08282-0.13801)^{2} \\
+(0.04948-0.49487)^{2}+(0.010206-0.10206)^{2} \\
+(0.04-0.1)^{2}
\end{array} \\
& \sqrt{=\sqrt{0.31706}=0.56308}
\end{aligned}
$$

The distance between the weighted score of each alternative to the ideal negative solution is calculated based on equation:

$$
\begin{aligned}
& D_{i}^{+}=\sqrt{\sum_{i=1}^{n}\left(Y_{i}^{+}-Y_{i j}\right)^{2}} \\
& D_{1}^{-}=\sqrt{\begin{array}{r}
(0.01905-0.0381)^{2}+(0.02760-0.02760)^{2} \\
+(0.04948-0.03299)^{2}+(0.06123-0.02041)^{2} \\
+(0.1-0.02)^{2}
\end{array}} \\
& =\sqrt{0.23782}=0.48766
\end{aligned}
$$$$
D_{2}^{-}=\sqrt{\begin{array}{c}
(0.05715-0.0381)^{2}+(0.05521-0.02760)^{2} \\
+(0.08247-0.03299)^{2} \\
+(0.04082-0.02041)^{2} \\
+(0.08-0.02)^{2}
\end{array}}
$$$$
=\sqrt{0.34961}=0.59127
$$

$D_{3}^{-}$

$$
\begin{aligned}
& \sqrt{(0.09525-0.0381)^{2}+(0.11043-0.02760)^{2}} \begin{array}{c}
+(0.049487-0.03299)^{2}+(0.06123-0.02041)^{2} \\
+(0.04-0.02)^{2}
\end{array} \\
& =\sqrt{0.35225}=0.59350
\end{aligned}
$$

$D_{4}^{-}$

$$
\begin{aligned}
& \sqrt{\begin{array}{c}
(0.01905-0.0381)^{2}+(0.13803-0.02760)^{2} \\
+(0.04948-0.03299)^{2} \\
+(0.010206-0.02041)^{2} \\
+(0.02-0.02)^{2}
\end{array}} \\
& =\sqrt{0.25262}=0.50261
\end{aligned}
$$

$$
D_{5}^{-}=\sqrt{\begin{array}{c}
(0.0381-0.0381)^{2}+(0.08282-0.02760)^{2} \\
+(0.03299-0.03299)^{2}+(0.02041-0.02041)^{2} \\
+(0.1-0.02)^{2}
\end{array}}
$$$$
=\sqrt{0.27018}=0.51978
$$$$
D_{6}^{-}=\sqrt{\begin{array}{c}
(0.05715-0.0381)^{2}+(0.08282-0.02760)^{2} \\
+(0.08247-0.03299)^{2} \\
+(0.02041-0.02041)^{2} \\
+(0.04-0.02)^{2}
\end{array}}
$$$$
=\sqrt{0.27871}=0.52792
$$$$
\begin{aligned}
& D_{7}^{-}=\sqrt{\begin{array}{c}
(0.0381-0.0381)^{2} \\
+(0.06598-0.03299)^{2} \\
+(0.05521-0.02760)^{2} \\
+(0.08-0.02)^{2}
\end{array}} \\
& =\sqrt{0.29638}=0.54440
\end{aligned}
$$$$
D_{8}^{-}=\sqrt{\begin{array}{c}
(0.01905-0.0381)^{2}+(0.05521-0.02760)^{2} \\
+(0.06798-0.03299)^{2}+(0.06123-0.02041)^{2} \\
+(0.04-0.02)^{2}
\end{array}}
$$$$
=\sqrt{0.23933}=0.48921
$$ 


\section{Decision Support System for Exemplary Teacher Selection in Elementary School using Topsis Method}

$$
\begin{aligned}
& D_{9}^{-}=\sqrt{\begin{array}{r}
(0.0381-0.0381)^{2}+(0.02760-0.02760)^{2} \\
+(0.08247-0.03299)^{2} \\
+(0.04082-0.02041)^{2} \\
+(0.02-0.02)^{2}
\end{array}} \\
& =\sqrt{0.20485}=0.45260 \\
& D_{10}^{-}= \\
& \begin{array}{c}
(0.03675-0.0381)^{2}+(0.08282-0.02760)^{2} \\
+(0.04948-0.03299)^{2}+(0.010206-0.02041)^{2} \\
+(0.04-0.02)^{2}
\end{array} \\
& =\sqrt{0.21511}=0.46379
\end{aligned}
$$

The proximity of each alternative to the ideal solution is calculated based on equations:

$$
\begin{aligned}
& V_{i}=\frac{D_{I}^{-}}{D_{I}^{-}+D_{I}^{+}} \\
& V_{1}=\frac{0.48766}{0.59560+0.48766}=\frac{0.48766}{1.08326}=0.45017 \\
& V_{2}=\frac{0.59127}{0.58910+0.59127}=\frac{0.59127}{1.18037}=0.50091 \\
& V_{3}=\frac{0.59350}{0.67361+0.59350}=\frac{0.59350}{1.26711}=0.46838 \\
& V_{4}=\frac{0.50261}{0.57842+0.50261}=\frac{0.50261}{1.08103}=0.46493 \\
& V_{5}=\frac{0.51978}{0.63614+0.51978}=\frac{0.51978}{1.15592}=0.44966 \\
& V_{6}=\frac{0.52792}{0.56058+0.52792}=\frac{0.52792}{1.12352}=0.469880 \\
& V_{7}=\frac{0.54440}{0.60406+0.54440}=\frac{0.54440}{1.14846}=0.47402 \\
& V_{8}=\frac{0.48921}{0.55122+0.48921}=\frac{0.48921}{1.04043}=0.47019 \\
& V_{9}=\frac{0.45260}{0.49029+0.45260}=\frac{0.45260}{0.94289}=0.48001 \\
& V_{10}=\frac{0.46379}{0.56308+0.46379}=\frac{0.46379}{1.02687}=0.45165
\end{aligned}
$$

From the score of V obtained the score of V5 $=0.44966$ has the smallest score and V6 $=0.469880$ has the greatest score as the best teacher performance.

\section{B. Implementation}

Decision support system application determined exemplary teacher performance by using Microsoft Excel as follows:

1. Alternatives and criteria of the running system.

Table-IX: Alternative and criteria

\begin{tabular}{|l|l|l|l|l|l|l|}
\hline & $\boldsymbol{C 1}$ & $\boldsymbol{C 2}$ & $\boldsymbol{C 3}$ & $\boldsymbol{C 4}$ & $\boldsymbol{C 5}$ & ROOT \\
\hline $\boldsymbol{A 1}$ & 1 & 1 & 3 & 3 & 5 & 2 \\
\hline $\boldsymbol{A 2}$ & 3 & 2 & 5 & 2 & 4 & 2 \\
\hline $\boldsymbol{A 3}$ & 5 & 4 & 3 & 3 & 2 & 2 \\
\hline $\boldsymbol{A 4}$ & 1 & 5 & 3 & 5 & 1 & 2 \\
\hline $\boldsymbol{A 5}$ & 2 & 3 & 2 & 1 & 5 & 2 \\
\hline $\boldsymbol{A 6}$ & 3 & 3 & 5 & 1 & 2 & 2 \\
\hline $\boldsymbol{A 7}$ & 2 & 2 & 4 & 3 & 4 & 2 \\
\hline $\boldsymbol{A} \boldsymbol{8}$ & 1 & 2 & 4 & 3 & 2 & 2 \\
\hline $\boldsymbol{A 9}$ & 2 & 1 & 5 & 2 & 1 & 2 \\
\hline $\boldsymbol{A 1 0}$ & 2 & 3 & 3 & 5 & 2 & 2 \\
\hline
\end{tabular}

2. Calculated the normalized decision matrix (R)

Table-X: Matrix Process

\begin{tabular}{|l|l|l|l|l|l|l|}
\hline & $\boldsymbol{C 1}$ & $\boldsymbol{C 2}$ & $\boldsymbol{C 3}$ & $\boldsymbol{C 4}$ & $\boldsymbol{C 5}$ & $\boldsymbol{R O O T}$ \\
\hline $\boldsymbol{A 1}$ & 1 & 1 & 3 & 3 & 5 & 2 \\
\hline $\boldsymbol{A} \boldsymbol{2}$ & 3 & 2 & 5 & 2 & 4 & 2 \\
\hline $\boldsymbol{A 3}$ & 5 & 4 & 3 & 3 & 2 & 2 \\
\hline $\boldsymbol{A 4}$ & 1 & 5 & 3 & 5 & 1 & 2 \\
\hline
\end{tabular}

Published By

5. Score for Matrix Y negative (D-)

\begin{tabular}{|l|l|l|l|l|}
\hline 0.15 & 0.25 & 0.2 & 0.2 & 0.2 \\
\hline
\end{tabular}

Table-XIII: Score of Matrix Y

\begin{tabular}{|l|l|l|l|l|}
\hline 0.01905 & 0.027608 & 0.049487 & 0.061237 & 0.1 \\
\hline 0.05715 & 0.055216 & 0.082479 & 0.040825 & 0.08 \\
\hline 0.09525 & 0.110432 & 0.049487 & 0.061237 & 0.04 \\
\hline 0.01905 & 0.138039 & 0.049487 & 0.102062 & 0.02 \\
\hline 0.0381 & 0.082824 & 0.032991 & 0.020412 & 0.1 \\
\hline 0.05715 & 0.082824 & 0.082479 & 0.020412 & 0.04 \\
\hline 0.0381 & 0.055216 & 0.065983 & 0.061237 & 0.08 \\
\hline 0.01905 & 0.055216 & 0.065983 & 0.061237 & 0.04 \\
\hline 0.0381 & 0.027608 & 0.082479 & 0.040825 & 0.02 \\
\hline 0.0381 & 0.082824 & 0.049487 & 0.102062 & 0.04 \\
\hline
\end{tabular}

6. Calculated distance ideal positive solution (D+) and

Table-XIV: Ideal positive solution (D+) and Negative (D-)

\begin{tabular}{|c|l|l|c|l|}
\hline D & POSITIVE & NEGATIVE & ROOT + & ROOT - \\
\hline D1 & 0.020757 & 0.017324 & 0.144072 & 0.131621 \\
\hline D2 & 0.012461 & 0.021184 & 0.111631 & 0.145548 \\
\hline D3 & 0.007117 & 0.029067 & 0.084364 & 0.17049 \\
\hline D4 & 0.013295 & 0.032683 & 0.115303 & 0.180786 \\
\hline
\end{tabular}




\begin{tabular}{|l|l|l|l|l|} 
D5 & 0.015431 & 0.019816 & 0.12422 & 0.140771 \\
\hline D6 & 0.014767 & 0.018945 & 0.12152 & 0.137642 \\
\hline D7 & 0.012465 & 0.019004 & 0.111645 & 0.137855 \\
\hline D8 & 0.018205 & 0.013115 & 0.134926 & 0.114523 \\
\hline D9 & 0.025611 & 0.011083 & 0.160035 & 0.105277 \\
\hline D10 & 0.011003 & 0.022777 & 0.104897 & 0.150921 \\
\hline
\end{tabular}

7. Calculated preference score for every alternative

Table-XV: Alternative preference

\begin{tabular}{|c|c|c|c|c|}
\hline$D$ & $\begin{array}{l}\text { POSIT } \\
\text { IVE }\end{array}$ & $\begin{array}{l}\text { NEG } \\
A T I \\
V E\end{array}$ & $\begin{array}{l}R O O \\
T+\end{array}$ & $\begin{array}{l}\text { RO } \\
\text { OT }-\end{array}$ \\
\hline D1 & $\begin{array}{l}0.0207 \\
57\end{array}$ & $\begin{array}{l}0.01 \\
7324\end{array}$ & $\begin{array}{l}0.14 \\
4072\end{array}$ & $\begin{array}{l}0.13 \\
162 \\
1\end{array}$ \\
\hline D2 & $\begin{array}{l}0.0124 \\
61\end{array}$ & $\begin{array}{l}0.02 \\
1184\end{array}$ & $\begin{array}{l}0.11 \\
1631\end{array}$ & $\begin{array}{l}0.14 \\
554 \\
8\end{array}$ \\
\hline D3 & $\begin{array}{l}0.0071 \\
17\end{array}$ & $\begin{array}{l}0.02 \\
9067\end{array}$ & $\begin{array}{l}0.08 \\
4364\end{array}$ & $\begin{array}{l}0.17 \\
049\end{array}$ \\
\hline D4 & $\begin{array}{l}0.0132 \\
95\end{array}$ & $\begin{array}{l}0.03 \\
2683\end{array}$ & $\begin{array}{l}0.11 \\
5303\end{array}$ & $\begin{array}{l}0.18 \\
078 \\
6\end{array}$ \\
\hline D5 & $\begin{array}{l}0.0154 \\
31\end{array}$ & $\begin{array}{l}0.01 \\
9816\end{array}$ & $\begin{array}{l}0.12 \\
422\end{array}$ & $\begin{array}{l}0.14 \\
077 \\
1\end{array}$ \\
\hline D6 & $\begin{array}{l}0.0147 \\
67\end{array}$ & $\begin{array}{l}0.01 \\
8945\end{array}$ & $\begin{array}{l}0.12 \\
152\end{array}$ & $\begin{array}{l}0.13 \\
764 \\
2\end{array}$ \\
\hline D7 & $\begin{array}{l}0.0124 \\
65 \\
\end{array}$ & $\begin{array}{l}0.01 \\
9004 \\
\end{array}$ & $\begin{array}{l}0.11 \\
1645 \\
\end{array}$ & $\begin{array}{l}0.13 \\
785 \\
5 \\
\end{array}$ \\
\hline D8 & $\begin{array}{l}0.0182 \\
05 \\
\end{array}$ & $\begin{array}{l}0.01 \\
3115 \\
\end{array}$ & $\begin{array}{l}0.13 \\
4926 \\
\end{array}$ & $\begin{array}{l}0.11 \\
452 \\
3 \\
\end{array}$ \\
\hline$D^{9}$ & $\begin{array}{l}0.0256 \\
11\end{array}$ & $\begin{array}{l}0.01 \\
1083\end{array}$ & $\begin{array}{l}0.16 \\
0035\end{array}$ & $\begin{array}{l}0.10 \\
527 \\
7\end{array}$ \\
\hline$D 10$ & $\begin{array}{l}0.0110 \\
03\end{array}$ & $\begin{array}{l}0.02 \\
2777 \\
\end{array}$ & $\begin{array}{l}0.10 \\
4897 \\
\end{array}$ & $\begin{array}{l}0.15 \\
092 \\
1 \\
\end{array}$ \\
\hline
\end{tabular}

\begin{tabular}{|l|}
\hline $\begin{array}{l}(+)+ \\
(-)\end{array}$ \\
\hline 0.27 \\
5693 \\
\hline \\
0.25 \\
7179 \\
\hline 0.25 \\
4854 \\
\hline 0.29 \\
6089 \\
\hline 0.26 \\
4991 \\
\hline 0.25 \\
9162 \\
\hline 0.24 \\
9501 \\
\hline 0.24 \\
9448 \\
\hline 0.26 \\
\hline 5312 \\
\hline 0.25 \\
5817 \\
\hline
\end{tabular}

\begin{tabular}{|l|}
\hline $\begin{array}{l}\text { Resu } \\
l t\end{array}$ \\
\hline 0.47 \\
7419 \\
\hline \\
0.56 \\
5942 \\
\hline 0.66 \\
897 \\
\hline 0.61 \\
0578 \\
\hline \\
0.53 \\
123 \\
\hline \\
0.53 \\
1104 \\
\hline 0.55 \\
2525 \\
\hline 0.45 \\
9103 \\
\hline 0.39 \\
6804 \\
\hline 0.58 \\
9954 \\
\hline
\end{tabular}

8. Ideal Matrix Score Max, Min

Table-XVI: Max, Min ideal

\begin{tabular}{|c|c|c|c|c|c|}
\hline MAX & 0.0952 & 0.1380 & 0.0824 & 0.10206 & \\
& 5 & 39 & 79 & 2 & 0.1 \\
\hline MIN & 0.0381 & 0.0276 & 0.0329 & 0.02041 & \\
\hline KUADRAT & 2 & 2 & 2 & 2 & 0.02 \\
\hline
\end{tabular}

\section{Analysis of Research Results}

Test results applied the TOPSIS method with the calculation manually. Made rank every criteria weight so can be obtained alternative 6 selected as best exemplary teacher performance with score of $\mathrm{V} 6=0.469880$.

\section{CONCLUSION}

Based on the explanation above, the conclusion of this research is that the teacher's performance assessment with the teacher's performance criteria, education level, work discipline, work quality, length of work and obtained result was alternative 6 with score of 0.469880 stating teachers with good performance.
The author gives some suggestions that may be helpful in the development of this journal:

1. Need to be added more the other criteria so that the data obtained is more accurate.

2. TOPSIS can be used for teacher assessment, but until this time the author utilizes this method the author has not been able to implement it. So it is hoped that this research can continues to accelerate the assessment process.

3. As has been told in the previous chapter where the search method is the main cause in which the results of this research get the results as mentioned. Expected in next research about this method can find a better research method so as to be better.

\section{REFERENCES}

1. E. Turban, J. E. Aronson, and T.-P. Liang, "Decision Support Systems and Intelligent Systems," Decis. Support Syst. Intell. Syst., vol. 7, p. 867, 2007.

2. E. Turban, R. Sharda, and D. Delen, Decision Support and Business Intelligence Systems. Chapter 6 Artificial Neural Networks for Data Mining, vol. 8th. 2007.

3. M. Muslihudin, F. Triananingsih, and L. Anggraei, "Pembuatan Model Penilaian Indeks Kinerja Dosen Menggunakan Metode Fuzzy Simple Additive Weighting," SEMNASTEKNOMEDIA, vol. 5, no. 1, pp 25-30, 2017.

4. Undang-Undang RI, "Undang-undang Republik Indonesia Nomor 14 Tahun 2005 tentang Guru dan Dosen," Undang. RI, p. 54, 2005.

5. Maryam, Siti Siregar. 2015. Sistem Pendukung Keputusan Pemilihan Guru Teladan di SMA Era Utama Pancur Batu. Medan

6. https://www.ilmuskripsi.com/2016/05/jurnal-sistem-pendukung-keput usan_19.html

7. S. K. Patil and R. Kant, "A fuzzy AHP-TOPSIS framework for ranking the solutions of Knowledge Management adoption in Supply Chain to overcome its barriers," Expert Syst. Appl., vol. 41, no. 2, 2014.

8. Z. Yue, "TOPSIS-based group decision-making methodology in intuitionistic fuzzy setting," Inf. Sci. (Ny)., vol. 277, 2014.

9. R. A. Krohling, R. Lourenzutti, and M. Campos, "Ranking and comparing evolutionary algorithms with Hellinger-TOPSIS," Appl. Soft Comput. J., vol. 37, 2015.

10. R. A. Krohling and A. G. C. Pacheco, "A-TOPSIS - An approach based on TOPSIS for ranking evolutionary algorithms," in Jurnal TAM (Technology Acceptance Model) Volume 9, Nomor 1, Juli 2018 Hal. 36-42 p-ISSN : 2339-1103 e-ISSN : 2579-4221, Procedia Computer Science, 2015, vol. 55, pp. 308-317.

11. S. Başaran and Y. Haruna, "Integrating FAHP and TOPSIS to evaluate mobile learning applications for mathematics," in Procedia Computer Science, 2017, vol. 120, pp. 91-98.

12. I. Ertugrul and T. Oztas, "Business mobile-line selection in Turkey by using fuzzy TOPSIS , one of the multi-criteria decision methods,' Procedia - Procedia Comput. Sci., vol. 31, pp. 40-47, 2014.

13. R. Suhandi, L. Anggraeni, and M. Muslihudin, "Cara Penentuan Kelayakan Calon Kepala Desa Pada Desa Blitarejo Mengunakan Metode Simple Additive Weighting (SAW)," in KNSI 2016, 2016, pp. $11-13$

14. Guntur Perdana, Tri Widodo. 2013. Sistem Pendukung Keputusan Pemberian Beasiswa Kepada Peserta Didik Baru Menggunakan Metode TOPSIS. SEMANTIK 2013. Hal 265-272. Semarang.

15. Pangeran Manurung. 2010. Sistem Pendukung Keputusan Seleksi Penerima Beasiswa Dengan Metode Ahp Dan Topsis (Studi Kasus: FMIPA USU). Skripsi. Universitas Sumatera Utara.

16. Santoso Wibowo and Hepu Deng, Intelligent Decision Support for Criteria Weighting in Multicriteria Analysis for Evaluating and Selecting Cargo Ships under Uncertainty, Proceedings of the International MultiConference of Engineers and Computer Scientists (IMECS 2011), Vol.2, 2011.

17. Efraim Turban and. Jay E. Aronson. Decision Support Systems and. Intelligent Systems. 6th edition. Prentice Hall, Upper Saddle River, NJ, 2001 . 